\title{
ROTATIONALLY SYMMETRIC SOLUTIONS FOR SHALLOW MEMBRANE CAPS*
}

\author{
BY \\ R. W. DICKEY \\ University of Wisconsin, Madison
}

\begin{abstract}
We discuss the displacements and stresses in a shallow membrane cap with either displacement or stress prescribed on the boundary and subjected to a small applied pressure. It is shown that if the prescribed boundary stress is sufficiently large there is a rotationally symmetric solution and if the applied pressure is large there is a solution to the problem with zero displacement prescribed on the boundary. However, there are situations in which there are no rotationally symmetric solutions. One case is described in which there are no rotationally symmetric solutions unless the prescribed boundary stress or prescribed boundary displacement is large.
\end{abstract}

1. Introduction. The purpose of this paper is to find the displacements and stresses in a shallow membrane cap with displacement or stress prescribed on the boundary and subjected to a small gravitational force or normal pressure.

A small strain theory for the rotationally symmetric deformation of membrane caps has been obtained by Bromberg and Stoker [1], Reissner [2], and the author [3], [4]. In the appendix (Sec. 6) it is shown that the small strain, small pressure theory described in [3] and [4] can be written

$$
r^{2} \sigma_{r}^{\prime \prime}+3 r \sigma_{r}^{\prime}=\frac{E}{2}\left(Z^{\prime}\right)^{2}-\frac{E^{3}}{2}\left(\frac{G}{\sigma_{r}}\right)^{2},
$$

$('=d / d r)$ where $\sigma_{r}$ is the radial stress, $E$ is the Young's modulus, and $G$ is the known function

$$
G=\frac{1}{E h r} \int_{0}^{r} t P(t) d t
$$

with $h$ the thickness of the membrane and $P(r)$ the applied pressure. The undeformed membrane has the shape of the surface constructed by rotating the curve $Z=Z(r)$ $(0 \leq r \leq a)$ about the $Z$ axis. We will assume that $Z(r)$ has the form

$$
Z=C\left(1-\left(\frac{r}{a}\right)^{\gamma}\right), \quad 0 \leq r \leq a .
$$

The constant $C$ is the height of the membrane at the center and the shape of the membrane is determined by $\gamma$. For example, if $\gamma=1$, Eq. (1.3) describes a cone

${ }^{*}$ Received January 30, 1989. 
with altitude $C$ and base radius $a$. We will see in Sec. 2 that $\gamma=2$ corresponds to a spherical cap if $C$ is small.

Once $\sigma_{r}$ is determined from (1.1) the circumferential stress $\sigma_{\theta}$, the radial displacement $u$, and the displacement in the $Z$ direction $w$ are determined. In particular (cf. [3] or [4])

$$
\begin{gathered}
\sigma_{\theta}=\left(r \sigma_{r}\right)^{\prime} \\
u=\frac{r}{E}\left(\left(r \sigma_{r}\right)^{\prime}-\nu \sigma_{r}\right) \\
w=E \int_{r}^{a} \frac{G}{\sigma_{r}} d t
\end{gathered}
$$

where $\nu$ is the Poisson ratio. For the boundary conditions on (1.1) either stress is prescribed

$$
\sigma_{r}(a)=\sigma
$$

or radial displacement is prescribed (cf. (1.5))

$$
u(a)=\frac{a}{E}\left(a \sigma_{r}^{\prime}(a)+(1-\nu) \sigma_{r}(a)\right)=\mu .
$$

For both (1.7) and (1.8) we will need a regularity condition at $r=0$. The appropriate condition is discussed in Sec. 3.

Two special cases of (1.1) have been studied in detail: The plane membrane, i.e., $C=0$ (cf. [5-9]) and the spherical cap, i.e., $\gamma=2$ (cf. [1] and [10-11]). For the plane membrane it is known that under reasonable assumptions on the applied pressure Eq. (1.1) has a solution satisfying the boundary condition (1.7) for all $\sigma>0$ and it has a solution satisfying (1.8) with $\mu=0$. For the spherical cap it has been shown by Baxley [11] that Eq. (1.1) has a solution satisfying the boundary condition (1.8) with $\mu=0$ for all constant values of the pressure.

In this paper we treat Eq. (1.1) with $\gamma>1$ and, for simplicity, constant $P$. In Sec. 2 Eq. (1.1) is rewritten in dimensionless variables. In Sec. 3 an existence result is proved for Eq. (1.1) with boundary condition (1.7) if $\sigma$ is sufficiently large. In Sec. 4 an existence result is proved for Eq. (1.1) with boundary condition (1.8) if $\mu=0$ and either the applied pressure is sufficiently large or the membrane is sufficiently shallow. In Sec. 5 we discuss the special case $\gamma=\frac{4}{3}$. It is shown that if $\gamma=\frac{4}{3}$ and the pressure is large enough there is a solution to the stress problem for all $\sigma>0$ and to the displacement problem for all $\mu$. On the other hand if the pressure is small it is shown that the stress problem has no rotationally symmetric solution unless $\sigma$ is sufficiently large, and the displacement problem has no rotationally symmetric solution unless $\mu$ is sufficiently large. In particular there is no rotationally symmetric solution to the displacement problem with $\mu=0$. A different result has been obtained for the spherical cap $(\gamma=2)$. In this case it has been shown that the displacement problem $\mu=0$ has a solution for all values of the pressure (cf. [11]).

2. Dimensionless form of the equation. It is convenient to rewrite Eqs. (1.1) and (1.4)-(1.6) in dimensionless variables. Using Eq. (1.2) (with $P=$ constant) and (1.3) Eq. (1.1) takes the form

$$
\rho^{2} \ddot{\sigma}_{r}+2 \rho \dot{\sigma}_{r}=\frac{C^{2} \gamma^{2}}{2 a^{2}} \rho^{2 \gamma-2}-\frac{E P^{2} a^{2}}{8 h^{2}} \frac{\rho^{2}}{\sigma_{r}^{2}}
$$


$(\cdot=d / d \rho)$ where

$$
\rho=\frac{r}{a}
$$

Define two parameters

$$
\begin{gathered}
K=\left(\frac{E P^{2} a^{2}}{h^{2}}\right)^{1 / 3} \\
\lambda=\left(\frac{E C^{2} \gamma^{3} h}{P a^{4}}\right)^{1 / 3}=\frac{C \gamma h}{P a^{2}} K
\end{gathered}
$$

and a new dependent variable

$$
\sigma_{r}=K S_{r}
$$

In this notation Eq. (2.1) becomes

$$
\rho^{2} \ddot{S}_{r}+2 \rho \dot{S}_{r}=\frac{\lambda^{2}}{2} \rho^{2 \gamma-2}-\frac{\rho^{2}}{8 S_{r}^{2}} .
$$

The Eqs. (1.4)-(1.6) become

$$
\begin{gathered}
S_{\theta}=\frac{d}{d \rho}\left(\rho S_{r}\right) \\
U=\rho\left(\rho \dot{S}_{r}+(1-\nu) S_{r}\right) \\
W=\int_{\rho}^{1} \frac{t}{S_{r}} d t
\end{gathered}
$$

where $\sigma_{\theta}=K S_{\theta}, U=E u / a K$, and $W=2 E h K w / P a^{2}$. The boundary conditions (1.7), (1.8), and (1.9) become

$$
\begin{gathered}
S_{r}(1)=\frac{\sigma}{K}=S \\
U(1)=\dot{S}_{r}(1)+(1-\nu) S_{r}(1)=\frac{E \mu}{a K}=\Gamma .
\end{gathered}
$$

As a special case consider a spherical cap which is a portion of a sphere of radius $R$ and has base radius $a$. The exact equation for such a cap is

$$
Z=\sqrt{R^{2}-a^{2}}-\sqrt{R^{2}-r^{2}}=R\left(\sqrt{1-(a / R)^{2}}-\sqrt{1-(a \rho / R)^{2}}\right), \quad 0 \leq \rho \leq 1 .
$$

The requirement that the cap be shallow is simply the condition that $a / R \ll 1$. Thus (2.12) can be approximated by

$$
Z=\frac{a^{2}}{2 R}\left(1-\rho^{2}\right)
$$

It is a consequence of (2.13) that for the spherical cap $\gamma=2, C=a^{2} / 2 R$, and

$$
\lambda=\left(\frac{E a^{2} h}{P R^{3}}\right)^{1 / 3} .
$$

Eq. (2.6) with $\lambda$ given by (2.14) is exactly the problem treated in [10] and [11]. 
3. The stress problem. The differential Eq. (2.6) with boundary condition (2.10) can be rewritten as a nonlinear integral equation. There is no difficulty in showing that if $\gamma>1$ and $S_{r}$ satisfies the regularity condition $\lim _{\rho \rightarrow 0} \rho^{3} \dot{S}_{r}(\rho)=0$ then

$$
S_{r}=S-D\left(1-\rho^{2 \gamma-2}\right)+\frac{1}{8} \int_{\rho}^{1} \frac{1}{\eta^{3}}\left(\int_{0}^{\eta} \frac{\xi^{3} d \xi}{S_{r}^{2}}\right) d \eta
$$

where

$$
D=\frac{\lambda^{2}}{8 \gamma(\gamma-1)} .
$$

The problem is slightly simplified if we introduce a new dependent variable

$$
\Sigma=S_{r}+D\left(1-\rho^{2 \gamma-2}\right)
$$

so that (3.1) becomes

$$
\begin{aligned}
\Sigma & =S+\frac{1}{8} \int_{\rho}^{1} \frac{1}{\eta^{3}}\left(\int_{0}^{\eta} \frac{\xi^{3} d \xi}{\left[\Sigma-D\left(1-\xi^{2 \gamma-2}\right)\right]^{2}}\right) d \eta \\
& =T \Sigma .
\end{aligned}
$$

If $D=0$ (plane membrane) this equation has been solved by iteration when $S$ is sufficiently large (cf. [6], [9]) and the Schauder theorem has been used to prove existence for all $S>0$ (cf. [9]). The problem is more interesting if $D \neq 0$.

THEOREM. If $\gamma>1$ and $S-D>0$ Eq. (3.1) has a solution satisfying the bounds

$$
S-D<S_{r}<S+\frac{1-\rho^{2}}{64(S-D)^{2}} .
$$

Proof. Let $X$ be the Banach space consisting of continuous functions $f(\rho)$ on the interval $0 \leq \rho \leq 1$ with norm $\|f\|=\max _{0 \leq \rho \leq 1}|f(\rho)|$. Define a subset $A$ of $X$ by

$$
A=\left\{f \in X \mid m+D\left(1-\rho^{2 \gamma-2}\right) \leq f \leq M+D\left(1-\rho^{2 \gamma-2}\right)\right\}
$$

with

$$
\begin{gathered}
m=S-D \\
M=S+\frac{1-\rho^{2}}{64(S-D)^{2}} .
\end{gathered}
$$

It is immediate that $A$ is closed and convex. In order to apply the Schauder theorem (cf. [12]) it remains to show that $T$ (cf. (34)) is a compact operator mapping $A$ into itself.

Let $f \in A$ then (cf. (3.6))

$$
\begin{gathered}
T f \leq S+\frac{1}{8} \int_{\rho}^{1} \frac{1}{\eta^{3}}\left(\int_{0}^{\eta} \frac{\xi^{3} d \xi}{m^{2}}\right) d \eta=S+\frac{1-\rho^{2}}{64(S-D)^{2}} \\
=M \leq M+D\left(1-\rho^{2 \gamma-2}\right) ; \\
T f \geq S \geq S-D \rho^{2 \gamma-2}=S-D+D\left(1-\rho^{2 \gamma-2}\right)=m+D\left(1-\rho^{2 \gamma-2}\right) ;
\end{gathered}
$$

Thus $T$ maps $A$ into itself. 
In order to show $T$ is compact let $\left\{f_{n}\right\}$ be a sequence of functions in $A$. Then $\left\{T f_{n}\right\}$ and $\left\{\frac{d}{d \rho} T f_{n}\right\}$ are bounded independent of $n$. The boundedness of $\left\{T f_{n}\right\}$ follows from (3.9) and the boundedness of $\left\{\frac{d}{d \rho} T f_{n}\right\}$ is a consequence of

$$
\begin{aligned}
\left|\frac{d}{d \rho} T f_{n}\right| & =\frac{1}{8 \rho^{3}} \int_{0}^{\rho} \frac{\xi^{3} d \xi}{\left[f_{n}-D\left(1-\xi^{2 \gamma-2}\right)\right]^{2}} \\
& \leq \frac{1}{8 \rho^{3}} \int_{0}^{\rho} \frac{\xi^{3} d \xi}{m^{2}}=\frac{\rho}{32 m^{2}} \leq \frac{1}{32(S-D)^{2}} .
\end{aligned}
$$

The existence of a uniformly convergent subsequence of $\left\{T f_{n}\right\}$ follows from the Arzela lemma (cf. [13]). Thus $T$ is compact. The fact that $S_{r}$ satisfies the bounds (3.5) is a consequence of (3.3) and (3.6). Q.E.D.

If $S-D$ is sufficiently large it is possible to develop a convergent iterate scheme such as described in [6]. However, it is not clear that the scheme converges for small values of $S-D$. Of course the same difficulty was encountered in [6] and [9].

The theorem only proves existence of a solution to the stress problem (2.6) and (2.10) in the case $S-D>0$. We will see later (cf. Sec. 4) that, at least in the case in which $D$ is small, the solution of the displacement problem (2.6) and (2.11) with $\Gamma=0$ satisfies this condition. Nonetheless, it is plausible that there could be a solution to the stress problem with $S-D \leq 0$. In fact it will be shown in Sec. 5 that in the special case $\gamma=\frac{4}{3}$ and $D$ small there is a solution of the stress problem for all $S>0$.

Equation (3.1) was derived assuming the condition $\lim _{\rho \rightarrow 0} \rho^{3} \dot{S}_{r}(\rho)=0$. Equation (3.1) and the condition $S-D>0$ implies a stronger condition. We note that Eq. (3.4) implies

$$
\dot{\Sigma}=-\frac{1}{8 \rho^{3}} \int_{0}^{\rho} \frac{\xi^{3} d \xi}{\left[\Sigma-D\left(1-\xi^{2 \gamma-2}\right)\right]^{2}} .
$$

Thus $\Sigma$ is a decreasing function with $\Sigma(1)=S$ from which we conclude that $\Sigma(0) \geq S$. L'Hôpital's rule and the fact that $\Sigma(0) \geq S>D$ yields

$$
\dot{\Sigma}(0)=0 \text {. }
$$

Since Eq. (3.3) implies

$$
\dot{S}_{r}=\dot{\Sigma}+2(\gamma-1) D \rho^{2 \gamma-3}
$$

we find

$$
\lim _{\rho \rightarrow 0} \rho^{3-2 \gamma} \dot{S}_{r}(\rho)=2(\gamma-1) D .
$$

4. The displacement problem. The solution $\Sigma$ of Eq. (3.4) has the following properties:

(1) Let $\Sigma_{1}$ and $\Sigma_{2}$ be solutions of Eq. (3.4) corresponding to $S_{1}$ and $S_{2}$ and lying in $A_{1}$ and $A_{2}\left(A_{1}\right.$ and $A_{2}$ are the sets (3.6) corresponding to $S_{1}$ and $\left.S_{2}\right)$. If $S_{1}>S_{2}$ then $\Sigma_{1} \geq \Sigma_{2}$ for $0 \leq \rho \leq 1$ and $\Sigma_{1}>\Sigma_{2}$ when $\rho$ is sufficiently close to 1 .

(2) Let $\Sigma_{1}$ and $\Sigma_{2}$ be solutions of (3.4) corresponding to $S_{1}$ and $S_{2}$ and lying in $A_{1}$ and $A_{2}$. Then $\max _{0 \leq \rho \leq 1}\left|\Sigma_{1}(\rho)-\Sigma_{2}(\rho)\right|=\left|S_{1}-S_{2}\right|$.

The proofs of properties (1) and (2) are essentially identical to the proofs given in [6] for the properties of the radial stress in a plane circular membrane. It is a 
consequence of (2) that $\Sigma(\rho, S)$ (and $\dot{\Sigma}(\rho, S))$ is a continuous function of $S . S_{r}, S_{\theta}$, $U$, and $W$ are uniquely determined by $\Sigma$ (cf. (3.3), (2.7), (2.8), and (2.9)) and it follows that $S_{r}, S_{\theta}, U$, and $W$ are also continuous functions of $S$.

Equation (2.8) implies that

$$
U(1)=\dot{S}_{r}(1)+(1-\nu) S_{r}(1)=\dot{S}_{r}(1)+(1-\nu) S
$$

and Eq. (3.1) implies

$$
\dot{S}_{r}(1)=2(\gamma-1) D-\frac{1}{8} \int_{0}^{1} \frac{\xi^{3}}{S_{r}^{2}} d \xi
$$

Combining (4.1) and (4.2) we find

$$
U(1)=(1-\nu) S+2(\gamma-1) D-\frac{1}{8} \int_{0}^{1} \frac{\xi^{3}}{S_{r}^{2}} d \xi
$$

Equation (4.3) can be used to estimate $U(1)$. In particular the inequality (3.5) implies

$$
U(1) \leq(1-\nu) S+2(\gamma-1) D-\frac{1}{8} \int_{0}^{1} \frac{\xi^{3}}{\left[S+\frac{1-\xi^{2}}{64(S-D)^{2}}\right]^{2}} d \xi
$$

After an integration (4.4) can be written

$$
\begin{aligned}
U(1) \leq & (1-\nu) S+2(\gamma-1) D-4 \frac{(S-D)^{2}}{S} \\
& -256(S-D)^{4} \ln \left(\frac{S}{S+\frac{1}{64(S-D)^{2}}}\right) .
\end{aligned}
$$

If $D=0$ there are values of $S>0$ for which $U(1)<0$. Indeed, if $D=0$ and $S=.12$ the right side of $(4.5)$ is calculated to be approximately $-.238(\nu=0)$. Since the right side of (4.5) depends continuously on $D$ (for $D<S$ ) we conclude that when $D$ is sufficiently small, i.e., the membrane sufficiently shallow (or $P$ sufficiently large), there exist values of $S$ for which $U(1)<0$. It is easily seen that when $S$ is sufficiently large $U(1)>0$. Thus the continuity of $U(1)$ as a function of $S$ guarantees the existence of a value of $S$ such that $U(1)=0$ when $D$ is sufficiently small. Actually if $S_{r}$ is the unique solution of (3.1) satisfying the bounds (3.5) the value of $S$ for which $U(1)=0$ is unique. The proof of this fact is essentially identical to the proof of Theorem 5.4 in [6].

For the spherical membrane cap $(\gamma=2)$ Baxley [11] has shown that the displacement problem $U(1)=0$ has a solution for all $D>0$. However, it will be shown in Sec. 5 that this is not true for all $\gamma$. It will be shown that if $\gamma=\frac{4}{3}$ Eq. (2.6) with boundary conditions $(3.15)$ and $U(1)=0$ (cf. (2.11)) has no solution unless $\lambda^{2}$ is sufficiently small. This does not mean that the membrane cap has no solution-only that the solution, if it exists, is not rotationally symmetric. 
5. Special case $\gamma=\frac{4}{3}$. Callegari, Keller, and Reiss [8] have shown the equation governing the behavior of a plane circular membrane under constant pressure can be reduced to an autonomous system by an appropriate change of variable. The existence of a unique tensile solution $\left(S_{r}>0\right)$ and infinitely many compressive solutions $\left(S_{r}<0\right)$ follows from a phase plane diagram for this autonomous system. For the membrane cap with $\gamma=\frac{4}{3}$ it is also possible to reduce Eq. (2.6) to an autonomous system. The phase plane diagram for the membrane cap is more complicated than the diagram for the plane membrane and yields different results when $D$ is large $(P$. is small).

If $\gamma=\frac{4}{3}$ Eq. (2.6) becomes

$$
\rho^{2} \ddot{S}_{r}+3 \rho \dot{S}_{r}=\frac{\lambda^{2}}{2} \rho^{2 / 3}-\frac{\rho^{2}}{8 S_{r}^{2}} .
$$

Instead of the boundary value problem for $(5.1)$ we consider the related initial value problem

$$
\begin{gathered}
\rho^{2} \ddot{w}+3 \rho \dot{w}=\frac{\lambda^{2}}{2} \rho^{2 / 3}-\frac{\rho^{2}}{8 w^{2}} \\
w(0, b)=b>0 \\
\lim _{\rho \rightarrow 0} \rho^{1 / 3} \dot{w}(\rho, b)=\frac{2}{3} D .
\end{gathered}
$$

The condition (5.3b) is simply the regularity condition (3.15). The object is to choose $b$ so that either $w(1, b)=S$ (stress problem) or $\dot{w}(1, b)+(1-\nu) w(1, b)=\Gamma$ (displacement problem). It is convenient to introduce the change of dependent variable

$$
A=w / b
$$

and the change of independent variable

$$
t=\rho / b^{3 / 2} .
$$

In these new variables Eq. (5.2) becomes

$$
t^{2} A^{\prime \prime}+3 t A^{\prime}=\frac{\lambda^{2}}{2} t^{2 / 3}-\frac{t^{2}}{8 A^{2}}
$$

$('=d / d t)$. The initial conditions are

$$
\begin{gathered}
A(0)=1 \\
\lim _{t \rightarrow 0} t^{1 / 3} A^{\prime}(t)=\frac{2 D}{3} .
\end{gathered}
$$

If $A(t)$ is the solution of (5.6) satisfying the initial conditions $(5.7)$ then $w(\rho, b)=$ $b A\left(\rho / b^{3 / 2}\right)$ satisfies Eq. (5.2) and the initial conditions (5.3).

We will reduce (5.6) to a first order system by introducing the change of variable (cf. [8])

$$
\begin{aligned}
& \xi=t^{-2 / 3} A \\
& \eta=t^{1 / 3} A^{\prime} .
\end{aligned}
$$


There is no difficulty in showing that $\xi$ and $\eta$ satisfy the equations

$$
\begin{gathered}
\xi^{\prime}=\frac{1}{t}\left(\eta-\frac{2}{3} \xi\right) \\
\eta^{\prime}=\frac{1}{t}\left(\frac{-8 \eta}{3}-\frac{1}{8 \xi^{2}}+\frac{\lambda^{2}}{2}\right) .
\end{gathered}
$$

Combining (5.9) and (5.10) we see that $\eta(\xi)$ satisfies the first order autonomous equation

$$
\frac{d \eta}{d \xi}=\frac{-\frac{8 \eta}{3}-\frac{1}{8 \xi^{2}}+\frac{\lambda^{2}}{2}}{\eta-\frac{2 \xi}{3}}
$$

The initial condition on (5.11) is obtained from (5.7) and (5.8). Thus we find

$$
\begin{gathered}
\lim _{t \rightarrow 0} t^{2 / 3} \xi(t)=\lim _{t \rightarrow 0} A(t)=1 \\
\lim _{t \rightarrow 0} \eta(t)=\lim _{t \rightarrow 0} t^{1 / 3} A^{\prime}(t)=\frac{2 D}{3} .
\end{gathered}
$$

Equation (5.12) implies that $\lim _{t \rightarrow 0} \xi(t)=\infty$, so that

$$
\lim _{\xi \rightarrow \infty} \eta(\xi)=\frac{2 D}{3} \text {. }
$$

In order to draw the phase plane diagram for (5.11) we must first find the singularities. These singularities are simply those points which satisfy both

$$
\begin{gathered}
\eta-\frac{2 \xi}{3}=0 \\
-\frac{8 \eta}{3}-\frac{1}{8 \xi^{2}}+\frac{\lambda^{2}}{2}=0 .
\end{gathered}
$$

This pair of equations is easily reduced to the cubic

$$
\xi^{3}-\frac{9 \lambda^{2}}{32} \xi^{2}+\frac{9}{128}=0
$$

If $\lambda^{2}<\left(\frac{64}{3}\right)^{1 / 3}$ Eq. (5.16) has only one real root and this root is negative. If $\lambda^{2}>\left(\frac{64}{3}\right)^{1 / 3}$ Eq. (5.16) has three real roots-two are positive and one negative. In the special case $\lambda^{2}=\left(\frac{64}{3}\right)^{1 / 3}$ there are two real roots-a double positive root and a negative root. The negative root corresponds to compressive solutions and the analysis of this case is similar to that in [8]. In the case $\lambda^{2}=\left(\frac{64}{3}\right)^{1 / 3}$ the positive root is

$$
\xi_{0}=\left(\frac{9}{64}\right)^{1 / 3}
$$

and the corresponding value of $\eta$ is

$$
\eta_{0}=\left(\frac{1}{24}\right)^{1 / 3}
$$

It may be verified that this is an unstable nodal point (cf. [13]). 


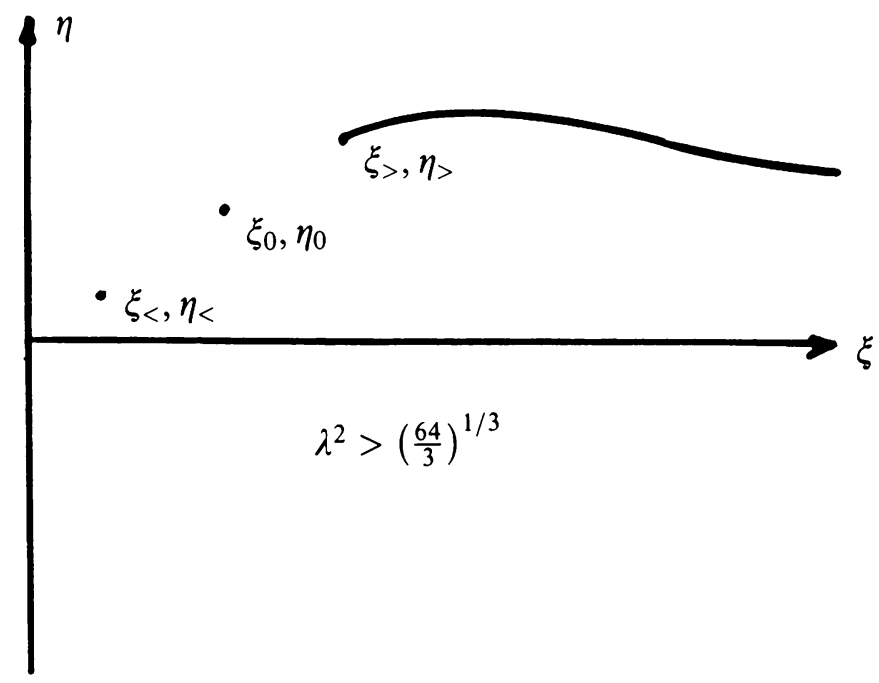

FIG. 1

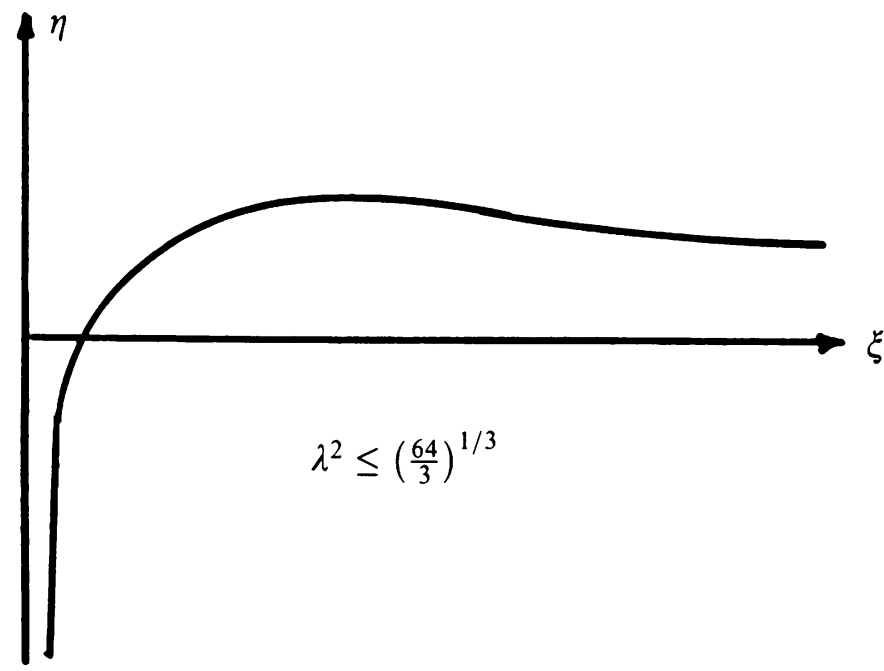

FIG. 2

If $\lambda^{2}<\left(\frac{64}{3}\right)^{1 / 3}$ Eq. (5.16) has one positive root $\xi_{>}$lying to the right of $\xi_{0}$ and one positive root $\xi_{<}$lying to the left of $\xi_{0}$, i.e., $\xi_{>}>\xi_{0}$ and $\xi_{<}<\xi_{0}$. The corresponding values of $\eta$ are $\eta_{>}=2 \xi_{>} / 3$ and $\eta_{<}=2 \xi_{<} / 3$. The singular point $\left(\xi_{>}, \eta_{>}\right)$is a stable nodal point while the singular point $\left(\xi_{<}, \eta_{<}\right)$is an unstable nodal point. The trajectory which satisfies the initial data (5.14) is drawn in Fig. 1. In the case $\lambda^{2} \leq\left(\frac{64}{3}\right)^{1 / 3}$ there are no stable equilibria in the right half-plane (in fact no equilibria if $\lambda^{2}<\left(\frac{64}{3}\right)^{1 / 3}$ ) and the trajectory corresponding to the initial data (5.14) is drawn in Fig. 2.

It is easily verified that if $\xi\left(1 / b^{3 / 2}\right)=S$ then $w(1, b)=S$ and if $\eta\left(1 / b^{3 / 2}\right)+$ $(1-\nu) \xi\left(1 / b^{3 / 2}\right)=\Gamma$ then $\dot{w}(1, b)+(1-\nu) w(1, b)=\Gamma$. Thus the solution of the 
stress problem is determined by the intersection of the line $\xi=S$ and the trajectory in Figs. 1 or 2 . Similarly the solution of the displacement problem is determined by the intersection of the line $\eta+(1-\nu) \xi=\Gamma$ and the trajectory in Figs. 1 and 2 .

We conclude that if $\gamma=\frac{4}{3}$ and $\lambda^{2} \leq\left(\frac{64}{3}\right)^{1 / 3}(P$ large) then the stress problem has a unique tensile solution for all $S>0$ and the displacement problem has a unique tensile solution for all $\Gamma$. If $\lambda^{2}>\left(\frac{64}{3}\right)^{1 / 3}(P$ small $)$ there is no solution to the stress problem unless (cf. (5.17) and (5.18))

$$
S>\xi_{>}>\xi_{0}
$$

and no solution to the displacement problem unless

$$
\Gamma>\eta_{>}+(1-\nu) \xi_{>}>\eta_{0}+(1-\nu) \xi_{0} .
$$

Unlike the spherical cap if $\gamma=\frac{4}{3}$ and $\lambda^{2}>\left(\frac{64}{3}\right)^{1 / 3}$ there is no rotationally symmetric solution of the problem $\Gamma=U(1)=0$.

6. Appendix. The equation governing the small strain behavior of a shallow cap under a gravitational force is (cf. [3])

$$
r^{2} T^{\prime \prime}+3 r T^{\prime}=\frac{\left(Z^{\prime}\right)^{2}}{2}+\nu(r G)^{\prime} \frac{G}{T}-\frac{1}{2}\left(\frac{G}{T}\right)^{2}
$$

$\left({ }^{\prime}=d / d r\right)$ where

$$
T=\sigma_{r} / E
$$

and the rest of the quantities are defined in Sec. 1. Rewriting Eq. (6.1) in terms of the radial stress we find

$$
r^{2} \sigma_{r}^{\prime \prime}+3 r \sigma_{r}^{\prime}=\frac{E\left(Z^{\prime}\right)^{2}}{2}+\nu E^{2}(r G)^{\prime} \frac{G}{\sigma_{r}}-\frac{E^{2}}{2}\left(\frac{G}{\sigma_{r}}\right)^{2} .
$$

We are looking for solutions of (6.3) which have the property that as the applied force $P \rightarrow 0(G \rightarrow 0)$, and the applied boundary stress $\sigma \rightarrow 0$ or boundary displacement $\mu \rightarrow 0$ then $\sigma_{r} \rightarrow 0$. It is a consequence of (6.3) (cf. [3]) that $G / \sigma_{r} \rightarrow Z^{\prime} / E$, i.e., $G / \sigma_{r} \nrightarrow 0$ as $P \rightarrow 0$. Thus, for small pressure, Eq. (6.3) can be replaced by (1.1).

In [3] and [4] it is shown that Eq. (1.1) also governs the small strain behavior of a shallow cap under normal pressure (pressure normal to the undeformed surface) and under hydrostatic pressure (pressure normal to the deformed surface).

\section{REFERENCES}

[1] E. Bromberg and J. J. Stoker, Non-linear theory of curved elastic sheets, Quart. Appl. Math. 3, 246-265 (1945/46)

[2] E. Reissner, Rotationally symmetric problems in the theory of thin elastic shells, 3rd U.S. Math. Congress of Applied Mechanics, 51-69, (1958)

[3] R. W. Dickey, Membrane caps, Quart. Appl. Math. 45, 697-712 (1987); Erratum, Quart. Appl. Math. 46, 192 (1988)

[4] R. W. Dickey, Membrane caps under hydrostatic pressure, Quart. Appl. Math. 46, $95-104$ (1988)

[5] H. Hencky, Über de Spannungszustand in kreisrunden Platten, Z. Math. Phys. 63, 311-317 (1915)

[6] R. W. Dickey, The plane circular elastic surface under normal pressure, Arch. Rational Mech. Anal. 26, 219-236 (1967)

[7] A. J. Callegari and E. L. Reiss, Non-linear boundary value problems for the circular membrane, Arch. Rational Mech. Anal. 31, 390-400 (1968) 
[8] A. J. Callegari, H. B. Keller, and E. L. Reiss, Membrane buckling. a study of solution multiplicity, Comm. Pure Appl. Math. 24, 499-527 (1971)

[9] H. J. Weinitschke, On finite displacements of circular elastic membranes, Math. Methods Appl. Sci. 9, 76-98 (1987)

[10] M. A. Goldberg, An iterative solution for rotationally symmetric non-linear membrane problems, Internat J. Non-linear Mech. 1, 169-178 (1966)

[11] J. V. Baxley, A singular nonlinear boundary value problem: Membrane response of a spherical cap, SIAM J. Appl. Math. 48, 497-585 (1988)

[12] J. T. Schwartz, Non-linear Functional Analysis, Gordon and Breach, New York, 1969

[13] E. A. Coddington and N. Levinson, Theory of Ordinary Differential Equations, McGraw-Hill, New York, 1955 